Jairo Francisco de Souza

\title{
Uma abordagem heurística uni-objetivo para calibragem em meta-alinhadores de ontologias
}

Tese apresentada ao Programa de Pós-graduação em Informática do Departamento de Informática da PUC-Rio como requisito parcial para obtenção do título de Doutor em Informática

Orientador : Prof. Carlos José Pereira de Lucena Co-Orientador: Prof. Rubens Nascimento Melo

Rio de Janeiro

Junho de 2012 


\title{
Jairo Francisco de Souza
}

\section{Uma abordagem heurística uni-objetivo para calibragem em meta-alinhadores de ontologias}

Tese apresentada ao Programa de Pós-graduação em Informática do Departamento de Informática do Centro Técnico Científico da PUC-Rio como requisito parcial para obtenção do título de Doutor em Informática. Aprovada pela comissão examinadora abaixo assinada.

\author{
Prof. Carlos José Pereira de Lucena \\ Orientador \\ Departamento de Informática - PUC-Rio
}

Prof. Rubens Nascimento Melo

Co-Orientador

Departamento de Informática - PUC-Rio

Prof. Karin Koogan Breitman Departamento de Informática - PUC-Rio

Prof. Antonio Luz Furtado

Departamento de Informática - PUC-Rio

Prof. Sean Wolfgand Matsui Siqueira

Departamento de Informática Aplicada - UNIRIO

Prof. Jonice de Oliveira Sampaio Departamento de Ciência da Computação - UFRJ

Prof. José Eugenio Leal

Coordenador do Centro Técnico Científico - PUC-Rio 
Todos os direitos reservados. Proibida a reprodução total ou parcial do trabalho sem autorização da universidade, do autor e do orientador.

Jairo Francisco de Souza

Graduou-se em Informática na Universidade Federal de Juiz de Fora. Concluiu o Mestrado em Engenharia de Sistemas e Computação na área de Banco de Dados na Universidade Federal do Rio de Janeiro. Concursado em 2009 como professor do Departamento de Ciência da Computação na Universidade Federal de Juiz de Fora, onde é coordenador do MBA em Gestão de Projetos de TI e pesquisador do Núcleo de Pesquisa em Engenharia do Conhecimento (NEnC). Ministrou aulas em cursos de extensão da PUC-Rio, participou de projetos de pesquisa na PUC-Rio e no Núcleo de Pesquisa e Prática em Tecnologia (NP2Tec) da UNIRIO, publicou artigos e capítulos de livros.

Ficha Catalográfica

Souza, Jairo Francisco de

Uma abordagem heurística uni-objetivo para calibragem em meta-alinhadores de ontologias / Jairo Francisco de Souza; orientador: Carlos José Pereira de Lucena; co-orientador: Rubens Nascimento Melo. - Rio de Janeiro : PUC-Rio, Departamento de Informática, 2012.

v., 105 f: il. ; $29,7 \mathrm{~cm}$

1. Tese (Doutorado em Informática) - Pontifícia Universidade Católica do Rio de Janeiro, Departamento de Informática.

Inclui referências bibliográficas.

1. Informática - Tese. 2. Alinhamento de ontologias. 3. Meta-alinhamento. 4. Calibragem de pesos. 5. Algoritmo genético. I. Lucena, Carlos José Pereira de. II. Melo, Rubens Nascimento. III. Pontifícia Universidade Católica do Rio de Janeiro. Departamento de Informática. IV. Título. 


\section{Agradecimentos}

Ao meu orientador, professor Carlos Lucena, por aceitar esta orientação.

Aos meus co-orientadores, professores Sean Siqueira e Rubens de Melo, por estimularem o meu trabalho e por serem exigentes e críticos para que eu obtivesse o melhor resultado.

Ao CNPq e à PUC-Rio, pelos auxílios concedidos, sem os quais este trabalho não poderia ter sido realizado.

Ao professor Stênio Sã, por disponibilizar várias horas do seu tempo para ensinar e discutir esta pesquisa.

Aos professores Antonio Furtado, Jonice Oliveira, Karin Breitman e Stenio Sã, por aceitarem participar da banca de defesa deste trabalho.

Aos meus colegas de trabalho e familiares, que incentivaram e torceram pela conclusão deste trabalho. 


\section{Resumo}

Souza, Jairo Francisco de; Lucena, Carlos José Pereira de; Melo, Rubens Nascimento. Uma abordagem heurística uni-objetivo para calibragem em meta-alinhadores de ontologias. Rio de Janeiro, 2012. 105p. Tese de Doutorado - Departamento de Informática, Pontifícia Universidade Católica do Rio de Janeiro.

Ontologias são vistas como uma solução para o problema de interoperabilidade em muitas aplicações como integração de banco de dados, comércio eletrônico, serviços web e redes sociais. Contudo, ao utilizar diferentes ontologias, persiste o problema de incompatibilidade semântica entre os modelos. Dentre os desafios para o campo de alinhamento de ontologia, está a seleção de alinhadores e a auto-configuração desses alinhadores. Este trabalho tem por objetivo tratar o problema de meta-alinhamento de ontologias utilizando métodos heurísticos para calibragem de alinhadores escolhidos sem detrimento do desempenho do sistema. Para avaliar esta proposta em comparação a outras propostas da literatura, optou-se por utilizar um benchmark para alinhamentos de ontologias amplamente utilizado por pesquisadores desse campo. A comparação entre as propostas é realizada com base em medidas de qualidade de alinhamentos sugeridas pelo benchmark. Além da avaliação quantitativa proposta pelo benchmark, é realizada uma comparação qualitativa em relação às características desejáveis de meta-alinhadores propostas na literatura.

\section{Palavras-chave}

Alinhamento de ontologias; Meta-alinhamento; Calibragem de pesos; Algoritmo genético; 


\section{Abstract}

Souza, Jairo Francisco de; Lucena, Carlos José Pereira de (Advisor); Melo, Rubens Nascimento (Co-advisor). A single goal heuristic approach to solve tuning in ontology meta-matching. Rio de Janeiro, 2012. 105p. DSc Thesis - Departamento de Informática, Pontifícia Universidade Católica do Rio de Janeiro.

Ontologies are seen as a solution for many applications such as database integration, e-commerce, web services and social networks. However, the use of distinct ontologies does not solve the semantic interoperability problem among models. Matcher selection and self-configuring are challenges from the ontology matching field. This work aims to treat the ontology metamatching problem using heuristic methods to fast tune a set of matchers. To evalutate this proposal, a well-kown benchmark for ontology matching is used. The comparison among meta-matching approaches includes the benchmark evaluation metrics and qualitative metrics for meta-matching proposed in the literature.

\section{Keywords}

Ontology matching; Meta-matching; Self-tuning; Genetic Algorithm; 


\section{Sumário}

1 Introdução $\quad 11$

1.1 Motivação 13

$\begin{array}{lll}1.2 & \text { Objetivos } & 16\end{array}$

1.3 Enfoque de solução e método de avaliação da pesquisa 16

$\begin{array}{lll}1.4 & \text { Organização da tese } & 18\end{array}$

2 Meta-alinhamento de ontologias $\quad 19$

$\begin{array}{ll}2.1 \text { Definições } & 19\end{array}$

$\begin{array}{ll}2.2 & \text { Algoritmos genéticos } \\ 2.3 & 24\end{array}$

2.3 Meta-alinhadores 27

2.4 Abordagens heurísticas para meta-alinhamento 29

3 Abordagem proposta para calibragem de alinhadores $\quad 35$

3.1 Descrição do problema 36

$\begin{array}{lll}3.2 & \text { Representação da solução } & 36\end{array}$

$\begin{array}{lll}3.3 & \text { Construtor das soluções iniciais } & 39\end{array}$

3.4 Operadores de reprodução e mutação 41

3.5 Intensificação da solução 44

3.6 Construção da nova geração 45

3.7 Demonstração do comportamento da abordagem 46

$4 \quad$ Implementação do meta-alinhador $\quad 53$

4.1 Arquitetura da ferramenta 53

4.2 Módulo de composição $\quad 54$

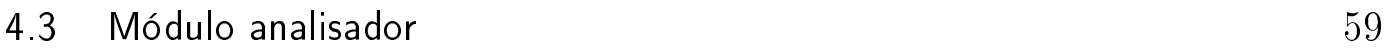

4.4 Módulo de persistência 63

5 Avaliação do meta-alinhador $\quad 64$

5.1 Descrição dos testes 64

5.2 Configuração utilizada para realização dos testes 67

$\begin{array}{lll}5.3 & \text { Resultados obtidos } & 69\end{array}$

$\begin{array}{lll}6 & \text { Análise dos resultados } & 75\end{array}$

6.1 Considerações quanto aos meta-alinhadores 75

6.2 Considerações quanto à base de teste 78

$\begin{array}{lll}6.3 & \text { Considerações finais } & 79\end{array}$

7 Conclusões $\quad 83$

$\begin{array}{lll}7.1 & \text { Contribuições } & 83\end{array}$

7.2 Limitações 84

7.3 Trabalhos futuros $\quad 85$

$\begin{array}{lr}\text { Referências Bibliográficas } & 88\end{array}$ 
$\begin{array}{ll}\text { A Algoritmos para medidas de similaridade de texto } & \mathbf{9 9}\end{array}$

$\begin{array}{ll}\text { A.1 lgualdade } & 99\end{array}$

$\begin{array}{lr}\text { A.2 Hamming } & 100\end{array}$

$\begin{array}{ll}\text { A.3 Levenshtein } & 100\end{array}$

A.4 Jaro-Winkler 101

A.5 Damerau-Levenshtein 101

$\begin{array}{ll}\text { A.6 TFIDF } & 102\end{array}$

B Arquivos XML utilizados para configuração do sistema 103 


\section{Lista de figuras}

$\begin{array}{lll}1.1 & \text { Quantidade de artigos publicados por ano } & 14\end{array}$

2.1 Método de ponto de cruzamento único, cruzamento de dois pontos e cruzamento uniforme

3.1 Representação da equação $\left(F_{1} P_{4}+F_{2} P_{5}\right) P_{1}+F_{4} P_{2}+F_{5} P_{3}=K_{1} \quad 37$

3.2 Cromossomo com destaque para a posicão de cada nível 40

3.3 Exemplo de cromossomo com valores válidos para funções do primeiro nível

3.4 Exemplo de um cromossomo com valores válidos

3.5 Técnica da roleta para seleção de indivíduos (Gudwin \& von Zuben, 1998)

3.6 Exemplo de crossover

3.7 Como uma nova população é gerada

3.8 Demonstração da convergência do AG para a equação 3-10 49

3.9 Gráfico ampliado da convergência do AG para a equação 3-10 49

3.10 Demonstração da convergência do AG para a equação 3-11 50

3.11 Gráfico ampliado da convergência do AG para a equação 3-11 51

3.12 Demonstração da convergência do AG para a equação 3-11 52

4.1 Arquitetura da ferramenta 54

4.2 Exemplo de documento XML para descrição de funções compostas 55

4.3 Exemplo de documento XML com alinhamentos de entrada 56

5.1 Gráfico triangular com cobertura e precisão totais 71

6.1 Comparação entre os meta-alinhadores heurísticos 81 


\section{Lista de tabelas}

2.1 Processo de evolução versus resolução em um problema de otimização, adaptado de (Talbi, 2009)

4.1 Algoritmos de distância de edição $\quad 57$

4.2 Funções de similaridade disponíveis no GNoSIS+ 58

4.3 Conjunto de tuplas 61

5.1 Tabela com o resumo dos testes realizados 66

5.2 Resultados encontrados no benchmark 70

5.3 Média harmônica dos resultados encontrados no benchmark 71

5.4 Comparação dos resultados com o meta-alinhador GOAL $\quad 72$

5.5 Comparação dos resultados com o meta-alinhador MaSiMe 73

5.6 Comparação entre a média harmônica dos meta-alinhadores GNoSIS+, GAOM, GOAL e MaSiMe 